\title{
True story about HIV: theory of viral sequestration and reserve infection
}

This article was published in the following Dove Press journal:

HIVIAIDS - Research and Palliative Care

7 December 20II

Number of times this article has been viewed

\section{Simon Situma Barasa \\ Kenya Polytechnic University College, Nairobi, Kenya}

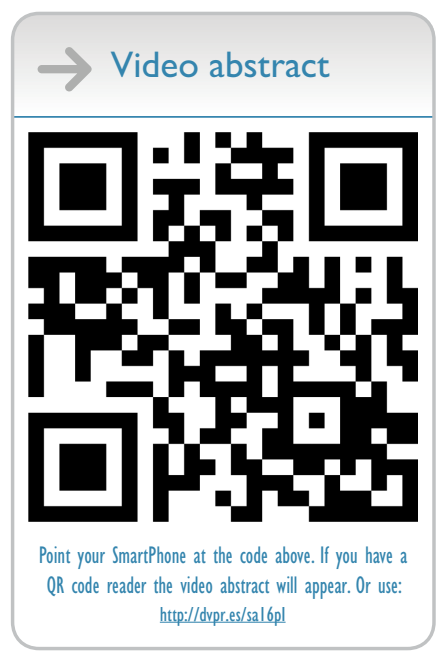

Correspondence: Simon Situma Barasa PO Box 330000100 , City Square, Nairobi, Kenya

Tel +25 4720837788

Email barasa_simon@yahoo.com
Abstract: Radical cure of infectious disease lies in the principle that the contagion is eliminated and its propagation within the body is stopped. By understanding the true mechanisms of human immunodeficiency virus (HIV) infection, effective cure is possible. Vertical research in HIV/ acquired immunodeficiency syndrome (AIDS) has produced much advanced data about its nature without discovering a true cure, implying that the infective concept may have been missed. Overall, current interventions have had a significant impact on the pandemic, but they definitely have not achieved a cure. Given that modern research has already provided almost all significant data on HIV/AIDS, this inevitably means that understanding of the HIV and AIDS mechanism in the human body and population is less than sufficient. This paper advances a new concept using the available scientific data in an attempt to open a new frontier in HIV research.

Keywords: human immunodeficiency virus, HIV, acquired immunodeficiency syndrome, AIDS, viral sequestration

\section{Introduction}

Human immunodeficiency virus (HIV) is a lentivirus, infection with which causes acquired immunodeficiency syndrome (AIDS) ${ }^{1,2}$ in humans, with progressive failure of the immune system, allowing life-threatening opportunistic infections and cancers to attack the body. Infection occurs by body fluid contact, ie, blood, semen, vaginal fluid, pre-ejaculate, and breast milk. The virus is present as both free virus particles and virus within infected cells in these fluids. The four main routes of transmission are unsafe sex, contaminated needles, breast milk, and perinatal transmission.

HIV infection in humans is classified as pandemic by the World Health Organization. From its recognition in 1981 through to 2006, AIDS killed more than 25 million people, with per capita infection of about $0.6 \%$ of the world's population. ${ }^{3}$ Estimated mortality from AIDS in 2009 has been put at 1.8 million persons, of whom approximately 260,000 were children. ${ }^{4}$ New infections in the same year were estimated at 2.6 million. The AIDS pandemic has particularly ravaged Sub-Saharan Africa, with disproportionately large numbers of deaths, slowing economic growth and exacerbating poverty levels. ${ }^{5}$ Use of antiretroviral therapy, particularly the so-called highly active antiretroviral therapy (HAART), reduces both mortality and morbidity associated with HIV infection. ${ }^{6}$ Expanded antiretroviral therapy programs since 2004 have reduced AIDS deaths and new infections in many parts of the world, ${ }^{4}$ although universal access to HAART and the optimal timing for initiation remain severely constrained, especially in Sub-Saharan Africa. Short-term and long-term adverse effects 
associated with use of HAART are of major clinical concern, impacting negatively on the quality of lives of patients under treatment. It is also noteworthy that, despite HAART, patients require more intensive health care and nutrition programs in the stages immediately after initiation of treatment. The overall cost of lifelong antiretroviral therapy is constrained by inadequate funding, with many countries relying on donor support, meaning sustainability of these programs cannot be guaranteed. Compliance with HAART can be compromised by adverse effects, opportunity, cost, culture, and ignorance, thus presenting the challenge of emergence of HIV strains resistant to currently available antiretroviral therapy. Preventive measures, such as awareness campaigns, prevention of mother to child transmission, and safe sex, have played a significant role in the fight against AIDS. However, these alone have not been demonstrated to decrease the rate of new infections significantly, because an estimated 2.6 million people were newly infected in $2009 .{ }^{4}$ Although the natural course of the AIDS epidemic might be expected, as is the case with other epidemics, to ameliorate with time, the high statistics of new infections and mortality show otherwise.

The principal target cells of HIV in the human body are CD4+ T lymphocytes, macrophages, and dendritic cells. ${ }^{7}$ AIDS is caused by a drastic reduction in CD4+ T lymphocytes to low critical levels whereby cell-mediated immunity is compromised and eventually lost. The CD4+ T lymphocyte count is diminished through direct viral killing of infected cells, increased rates of apoptosis in infected cells, and killing of infected CD4+ T cells by CD8 cytotoxic lymphocytes that recognize infected cells.

Untreated HIV infection in most individuals eventually develops into AIDS. ${ }^{8}$ Death is mostly due to opportunistic infections or malignancies associated with the progressive failure of the immune system. ${ }^{9}$ Individual variability of HIV progression to AIDS is attributed to viral, host, and environmental factors, with most cases occurring within 10 years of HIV infection. ${ }^{10,11}$ Antiretroviral therapy increases the life expectancy from an untreated mean of one year ${ }^{12}$ to an average survival time with antiretroviral therapy of more than 5 years as of $2005 .^{13}$

\section{Relevant virology}

HIV is a member of the genus Lentivirus ${ }^{14}$ and part of the Retroviridae family. ${ }^{15}$ It is transmitted as a single-stranded, positive-sense, enveloped virus. On entry into the target cell, the viral RNA genome is reverse-transcribed into doublestranded DNA by a virally encoded reverse transcriptase that is transported along with the viral genome in the virus particle. Importation of the resulting viral DNA into the host cell nucleus and integration into the cellular DNA by a virally encoded integrase and host cofactors follow. ${ }^{16} \mathrm{At}$ this stage, it has been proposed that the virus may become latent, allowing it and its host cell to avoid detection by the immune system for an indeterminate time. Alternatively, the virus is transcribed, producing new RNA genomes and viral proteins that are packaged and released from the cell as new virus particles that begin the replication cycle anew. Two subtypes of HIV have been characterized, ie, HIV-1 and HIV-2. HIV-1 was the one initially discovered and has been shown to be more virulent, more infective, and most widespread. HIV-2 is largely confined to West Africa due to its lower transmissibility. ${ }^{17,18}$

\section{Stages of HIV infection Primary stage}

Following exposure to an infective load of HIV, the primary stage establishes itself. Symptoms in most individuals $(80 \%-90 \%)$ during this stage include an influenza or mononucleosis-like illness called acute HIV infection, that most often includes fever, lymphadenopathy, pharyngitis, rash, myalgia, malaise, and mouth and esophageal sores, and may also include (albeit less commonly) headache, nausea and vomiting, an enlarged liver and/or spleen, weight loss, thrush, and neurological symptoms. Infected individuals may experience all, some, or none of these symptoms. The average duration of the illness is 28 days, and it usually lasts at least a week. ${ }^{19}$

This is the seeding stage when the virus establishes itself in the host system, and is marked with rigorous host immune resistance, as evident in first-line response symptoms that are generally similar to the body's reaction to many other infections. A period of rapid viral replication ensues, leading to an abundance of virus in the peripheral blood, with as many as several million virus particles reached per milliliter of blood. ${ }^{20}$

Acute viremia is accompanied by a marked drop in the numbers of circulating CD4+ T cells and is seen in virtually all patients. It is accompanied by activation of CD8+ T cells which kill HIV-infected cells and subsequent antibody production or seroconversion. The $\mathrm{CD} 8+\mathrm{T}$ cell response is thought to be important in controlling virus levels, which peak and then decline, as the CD4+ T cell counts rebound. $\mathrm{CD} 8+\mathrm{T}$ cell response has been linked to slower disease progression and a better prognosis, although it does not eliminate the virus. ${ }^{21}$ Attempts have been made to prevent infection at this stage using post exposure antiretroviral therapy for rape 
victims and children with a perinatal exposure risk, with encouraging results.

\section{Secondary (latency) stage}

The latency period lasts from 2 weeks to 20 years or more, depending on a variety of factors. Its hallmark is the absence of symptoms or few symptoms, depending on individuals. The viral load is initially reduced, but assumes a varying profile with time, generally increasing in geometric progression. HIV is active within the lymph nodes, which typically become persistently swollen in response to large amounts of virus that become trapped in the follicular dendritic cell network. ${ }^{22}$ The surrounding tissues that are rich in CD4+ $\mathrm{T}$ cells may also become infected, and viral particles accumulate both in infected cells and as free virus. Individuals who are in this stage remain infectious. During this time, CD4+ CD45RO+ T cells carry most of the proviral load. ${ }^{23}$ It has been shown that antiretroviral therapy started during this stage significantly improves survival, as compared with deferred therapy. ${ }^{24,25}$

\section{AIDS}

The final stage of HIV infection, ie, AIDS, is characterized by low CD4+ T cell counts with various opportunistic infections, cancers, and other conditions, leading to systems failure and death. Opportunistic or pathogenic infections and cancers in AIDS increase and worsen with falling CD4 counts, and fail to respond to known effective treatment because of reduced or absent cell-mediated immunity, which is normally ultimately responsible for any antimicrobial cure. Without treatment, patients will eventually die. HAART initiated early can lead to remarkable improvement in prognosis and even keep individuals healthy for indeterminate periods. Although HAART has been shown to reduce viral loads in the circulation to undetectable levels, it cannot eradicate the virus from the body, because its withdrawal leads to a rebound increase in viral load and progression to AIDS.

HIV controllers or long-term nonprogressors who retain high levels of CD4+ T cells without antiretroviral therapy and have a detectable viral load will eventually progress to AIDS without treatment. Elite suppressors, on the other hand, maintain CD4+ T cell counts and also have low or clinically undetectable viral load without antiretroviral treatment, ${ }^{26,27}$ suggesting they may have a genetic variation enabling them to control the virus even though they cannot eliminate it. HIV infects various cells, including CD4+ T cells, macrophages, and microglial cells. HIV-1 entry to macrophages and CD4+ $\mathrm{T}$ cells is mediated through interaction of virion envelope glycoproteins with the CD4 molecule on target cells and also with chemokine coreceptors. ${ }^{28}$

\section{Vaccination and therapy}

Immunomodulatory genes characterized to bear significance in control of HIV replication have led to a search for immunotherapy that may assist in the recovery of the immune system. ${ }^{29,30}$ It has been demonstrated that the immune response in chronic HIV is down regulated by a genetic mechanism in the face of persistent infection. This has led to investigation of whether reversing this down regulation by gene therapy would provide any advantage in the fight against HIV infection.

A vaccine that is a combination of two previously unsuccessful vaccine candidates (ALVAC-HIV and AIDSVAX) was reported in September 2009 to have resulted in a 30\% reduction in infections in a trial conducted in Thailand. ${ }^{31,32}$

HAART administered immediately after exposure, referred to as post exposure prophylaxis, is believed to reduce the risk of infection if started as quickly as possible. ${ }^{33}$ Early treatment of HIV-infected people with HAART protected $96 \%$ of partners from infection. ${ }^{34}$

A 40-year-old HIV-positive man was given a stem cell transplant as treatment for acute myelogenous leukemia, with the donor chosen for being homozygous for a CCR5$\Delta 32$ mutation that confers resistance to HIV infection. ${ }^{35,36}$ After 20 months without antiretroviral drug treatment, it was reported that HIV levels in the recipient's blood, bone marrow, and bowel were below the limit of detection. ${ }^{36}$ The virus remained undetectable for at least 3 years after the first transplant. ${ }^{37}$

\section{Pillars of faith Does the latency stage of HIV infection involve sequestration of virus beyond activity of the immune system?}

To answer this question, there is a need to look at the stages of HIV infection. In the primary stage, there is body fluid contact, ie, its introduction into the general body fluid and circulation compartment, with infection of immediately available target cells. Research has shown that the main routes of HIV transmission are unsafe sex, contaminated needles, breast milk, and perinatal transmission, all of which must involve fluid contact. This means the virus at this stage has to seed by rapid replication in the immediately available target cells in order to attain sufficient populations to cause effective infection elsewhere. That the infective viral load 
is very small compared with the levels of virus found at the peak of primary infection further demonstrates this point. The body mounts an aggressive immune response at this stage, as seen in the various symptoms of primary infection. This means that the immune system is able to recognize the virus at this stage and initiate nonspecific, then specific responses directed at containing the infection. The fact that primary symptoms abate after some time suggests that the immune system has indeed contained the primary infection, and secondary infection has a source other than the initial one. In patients given post exposure HAART, such as rape victims and neonates at risk of perinatal transmission in prevention of mother to child transmission, statistics show that effective transmission is drastically reduced, pointing to failure of primary infection to proceed to secondary infection. These groups do not contract long-term infection even though they have had exposure, meaning contamination of the general circulation compartment alone is not sufficient to confer chronic infection. HAART prevents the rapid initial replication needed to achieve populations required for effective chronic infection and, in concert with the aggressive immune responses; the virus is totally eliminated from the general circulation. Transmission of the virus from the mother to the child occurs in utero, intrapartum, or through breast feeding. In the absence of strategies for prevention of mother to child transmission, the transmission rate up to birth between the mother and child is about $25 \%{ }^{38}$ Using combination HAART and cesarean section, the risk is reduced to as low as $1 \%{ }^{38}$ Exclusive breast feeding and provision of extended prophylactic HAART to the infant are also effective in prevention of transmission. ${ }^{39}$ According to Joint United Nations Programme on HIV and AIDS estimates, 430,000 children were infected worldwide in 2008 (19\% of all new infections), primarily by this route, and 65,000 infections were avoided through the provision of antiretroviral prophylaxis to HIV-positive women. ${ }^{40}$

In a bone marrow transplant patient who was shown to be totally rid of the virus, only the bone marrow was changed to confer resistance to re-infection, even in the presence of other target cells in the general circulation, suggesting bone marrow is indeed a different and unique compartment in the process of HIV infection. The HIV was not removed from the patient's general circulation, dendritic cells, macrophages, or circulating long-life $\mathrm{T}$ memory lymphocytes. It was the reserve area of bone marrow that was changed, depriving the infection of a critical source of re-infection, thus allowing the immune system to clear the virus summarily from the "open" target cells. This principle is further supported by the fact that there exists a biological barrier in the bone marrow function of lymphocytogenesis. Compartmentalization in the bone marrow itself is well known, as is the biological blood-bone marrow barrier. The long period of latency of HIV infection, spanning on average 10 years to the onset of AIDS, represents the period when the reserve infection, although active, has not reached populations sufficient to decimate CD4+ T lymphocyte numbers. This means the virus has dodged the host defenses and is safely established, progressively replicating and releasing new particles into the general circulation. Latency means the virus cannot cause a sufficient decline in CD4+ T cells to cause AIDS, but it is still there in significant and increasing amounts, and yet it is able to avoid causing a remarkable host immune response on the scale seen at the primary infection stage. It has been suggested that the human immune modulation gene down regulates the response to a sustained viral presence, and that disabling this gene may reactivate aggressive immune reactivity that could control the progression to AIDS. It is apparent in this regard that HIV has found "safe" host cells from which it keeps re-infection of the whole host system going in spite of any immune mechanisms. Treatment using HAART may reduce this reserve infection, but being a competitive inhibition process, it cannot eliminate it. The virus sequestered in these safe cells is out of reach of the immune system and cannot be eradicated, whereas the virus released into the general circulation is controlled by HAART and eliminated by host immune mechanisms. This is why cessation of HAART leads to resurgence of viral loads and progression to AIDS without the initial symptoms of the primary stage. Thus, it can be inferred that there is no biological latency period in HIV infection, as suggested by proponents of the latent stage.

From the above, it is logical to conclude that the latency stage of HIV infection occurs when the immune system has subdued the viral infection in the general circulation, and chronic infection ensues because, by this time, the virus has already been sequestered in the bone marrow and established reserve infection.

\section{Does there exist such a unique compartment in human physiology where the HIV virus might hide in the form of reserve infection?}

There is in fact a physiological barrier between bone marrow and blood which prevents immature lymphocytes from entering the circulation. The bone marrow itself is composed 
of distinct biological compartments. This barrier is unique in that, unlike the blood-brain barrier, it restricts biological systems rather than chemical or structural systems. Because only mature lymphocytes can enter the circulation, it is possible that this is done to protect immature cells from some aspects in the general circulation. One such aspect could be the CD8 cytotoxic lymphocyte, which is credited with destruction of infected or malfunctioning cells, a process which results in the final eradication of any intracellular contagion and cancer. This system may also protect the HIV virus, which infects protected immature lymphocytes. Because lymphocytes are continuously produced to meet body demand according to biological feedback regulation, infection by the virus would not deplete all immature lymphocytes until such time when the viral load is overwhelmingly high, which can take a long time considering the dynamics of cell formation and the viral replication cycle, as well as the fact that not all infected cells are killed by apoptosis or direct viral action. It is proposed that depletion of CD4+ T lymphocytes occurs not only as a direct result of viral infection in the general circulation, but also as a result of reduced production due to infection of immature lymphocytes in the bone marrow. By transplantation of donor bone marrow resistant to infection, the patient described earlier was conferred with the ability to increase his CD4+ count despite presence of infection in other cells of the body. The new lymphocytes were not susceptible to infection, even when they entered circulation, while the older group of infected target cells was killed by apoptosis or CD8 action. The infection was thus eventually cleared by the immune system without the use of drugs.

The reason why it has proved difficult to develop an HIV vaccine can be explained in this context. Primary infection in a vaccinated individual still results in enough viral load to attain bone marrow sequestration, although it may be remarkably lower than the load in a non-vaccinated individual. The viral reserve in the bone marrow is able to avoid the ultimate step in elimination of contagions by staying out of the scope of CD8 cytotoxic lymphocytes, and probably other immune mechanisms necessary for elimination.

It can be concluded here that the bone marrow comprises a unique physiological compartment in which HIV is sequestered to maintain chronic infection in humans. This sequestration occurs after successful primary infection in the period before the immune system is able to subdue the initial infection. Subsequent infections attain levels sufficient to reach viral sequestration, hence re-infection with different chronic strains in the same patient.

\section{Is it possible to eliminate HIV infection using currently available antiretroviral therapy in the absence of reserve infection?}

Viral replication rates for primary HIV infection are not sustained into the latency stage. If such rates could be sustained, then the primary infection itself would be lethal and there would be no latency stage. In post exposure HAART, it is possible to eliminate the infection at the onset by prevention of progress to sequestration. In the bone marrow transplant HIV patient described earlier, elimination of viral reserve led to elimination of the virus elsewhere, even without further use of HAART. Vaccination can be possible if the immune system is able to prevent initial multiplication, dissemination, and sequestration of the virus. This can be demonstrated by the fact that some vaccine trials have shown a measure of success, and depending on the site and dosage of initial infection, the immune system may be able to eliminate the infection before sequestration.

It is logical to conclude that the immune system can eliminate HIV infection in the absence of reserve infection. HAART can prevent formation of reserve infection when given before viral sequestration has occurred, thus allowing the immune system to eliminate primary infection.

\section{What are the options that could be pursued to clear the said reserve infection and deal the virus the ultimate blow?}

Infection of any kind cannot be totally eliminated by drugs, which, regardless of their efficacy, act by competitive inhibition of cellular processes. A functional immune system is critical for the radical eradication of a contagion. This can be demonstrated by considering the progression of infection in immunocompromised patients. HIV elimination would similarly require a functional immune system even when effective drug therapy is available. Even though current HAART is effective in the control of infection in all target cells of the body, to achieve viral elimination, the cells bearing reserve infection in the bone marrow must be targeted and eliminated by the immune system without compromising the functional role of the blood-bone marrow barrier.

Research can be focused on achieving elimination or preventing the formation of reserve infection. Obviously if this is the point that has been missed in understanding of the infective circle of HIV, then herein lies the solution. Such a 
line of research is also likely to obtain more new information useful in medicine and science.

One of the questions to be addressed would be whether or not currently used drugs penetrate bone marrow in therapeutically significant amounts, and whether any changes or development can positively enhance activity within the viral reserve. Another line of research would be to determine the feasibility of implanting a remedy in the bone marrow, or breaching the bone marrow barrier for a period to allow full combat by the immune system. However, these will be subjects of future papers. It is possible to verify the veracity of these hypotheses by, for example, conducting animal-based research using similar infections such as simian immunodeficiency virus in monkeys.

\section{Infective story of HIV}

Upon entry of HIV into the system of an individual, the virus infects the immediately available target cells at the point of contact. It proceeds to replicate rapidly in these cells, with new viruses released and thus quickly infecting other available target cells. The increasing viral load disseminates via the circulation system, ie, the blood and lymphatic system, seeding and establishing more foci with replication. The initial rate of replication is high, and the resulting viral load increases in geometric progression. This leads to a rapid decline in CD4+ T lymphocytes as more cells are infected and die, probably because the feedback-mediated increase in production does not commence immediately or at a rate comparable with the rate of destruction. Also, at this point, the immune system recognizes the presence of infection and initiates a general nonspecific response, leading to the symptoms seen at primary infection. In the meantime, as viral replication continues, the immune system evaluates the virus successfully and initiates specific responses, which include production of specific antibodies (seroconversion) and surface antigen marking of infected cells for immune destruction. With the onset of the specific immune response, the infection is rapidly cleared and there is a rapid reduction in the viral population, with reduced infection of new target cells. There is also a rebound increase in CD4+ T lymphocytes as a result of increased feedback-mediated production as well as reduced infection of new cells.

However, before the immune system is ready to mount the specific response described above, the viral population peaks in the circulation and is widely disseminated in the body, with some becoming sequestered in the bone marrow compartment where the immune mechanism is unable to eliminate it. Although the immune system tries to eradicate the infection from the general circulation completely, the infection is maintained by constant supply of new virus from the reserve sequestered in the bone marrow. This is the chronic stage, and the peripheral viral load depends mainly on the dynamics of viral replication and immune action in the blood.

Immunomodulators downgrade the specific immune response against HIV over time, allowing the viral load to increase slowly and steadily, until it reaches a point when CD4+ T lymphocyte production cannot match destruction by infection. The CD4 count then slowly and steadily declines, along with a decline in cell-mediated immunity. When the CD4 count reaches a critical minimum at which cell-mediated immunity can no longer provide adequate protection, AIDS sets in.

Initiation of HAART immediately after infectious inoculation of HIV can abort chronic infection as long as it is done before viral replication has led to dissemination and sequestration of the virus in the bone marrow. In this case, the immune system completely eliminates any residual virus from the general circulation.

When bone marrow from a resistant individual is transplanted into an AIDS patient whose own bone marrow has been removed, the immune response is able to eliminate the virus from the body because of the absence of a sequestered reserve to maintain chronic infection.

It is impossible to achieve vaccination against HIV infection because, as with all vaccinations, a milder form of infection follows exposure in a vaccinated individual. This mild infection in the case of HIV is still sufficient to cause dissemination and bone marrow sequestration, hence chronic infection.

In discordant couples, when the resistant partner gets the first primary infection, that partner can transmit it to the susceptible mate during the initial period of infection but proceed to eliminate the virus from their own bodies because of absence of sequestration, while their partner develops chronic infection. Subsequent exposures lead to development of immune resistance in the resistant partner and do not cause primary infection due to absence of viral sequestration.

Re-infection in patients with chronic infection is possible because although initial infection provides protection against subsequent attacks, cell-mediated immunity in these individuals is usually reduced, allowing new infection to reach a population load adequate for sequestration in the 
bone marrow before it is subdued. Furthermore, a milder form of infection which usually follows exposure of immune individuals may still attain enough viral loads to cause sequestration.

Prevention of viral sequestration or elimination of reserve infection is the key to attaining a radical cure for HIV. Although post exposure HAART can prevent viral sequestration, its use in the general population would present enormous logistical challenges, rendering it impractical, in addition to being of no use in those with chronic infection. Therefore, the focus should be on elimination of reserve infection in infected individuals. Vaccines could be designed not just to protect against infection but to act as exposure alerts or alarm systems to enable effective post exposure HAART.

There cannot be any errors in these general propositions because the line of thinking is based on pure logic. However, variations in the results of specific aspects of this analysis are possible, although not in a manner that could be expected to change the theory in a radical way. Furthermore, should investigation radically change the flow of theory, it would still be credited for spurring that line of research.

\section{Summary}

Long-term HIV infection occurs when the virus is sequestered in "safe" cells in a unique body compartment where it forms a "reserve infection" and avoids eradication by the host immune system, and maintains general infection by continuous re-infection from this site. This unique compartment is the bone marrow. The latent stage of HIV infection is in fact an active stage in which reserve infection sequestered in "safe" cells of the bone marrow compartment continuously release new virus particles into the circulation, and AIDS occurs when the rate of viral production exceeds $\mathrm{T}$ cell replacement, leading to a gradual decline in CD4 count. Using available scientific data, knowledge, and logic, it is possible to demonstrate existence of these "safe" cells within the bone marrow as a unique physiological compartment, to show existence of a critical HIV reserve in these cells during chronic infection, and to show that absence or removal of the reserve infection leads to viral eradication, and thus provide the basis for new research along these lines. The explanations for various phenomena in HIV infection, such as discordant partners, re-infection, vaccine failure, and long periods of apparent latency, lie in the theory of critical reserve infection sequestered in the "safe" cells of the bone marrow. The human immune system can eliminate HIV infection from the general target cells of the body if viral sequestration is prevented or reserve infection is removed from its site of safe sequestration in the bone marrow.

This theory discredits the perception that long-term HIV infection occurs at the onset of primary infection and is maintained within the general target cells, with the host immune system and current drugs being unable to eliminate it effectively. That HIV maintains infection by passive latency, whereby some of the integrated viral DNA remains dormant within the nucleus of the host cell, retaining a capability to activate a full cycle of viral replication in the future. That no unique "safe" cells or compartment in the body where reserve HIV infection is sequestered away from active host immunity or drugs can be demonstrated using the available scientific data and logic. That it is not possible to achieve a radical cure of HIV infection by focusing research on the principle of safe cells for reserve HIV infection in the body. And that the human immune system cannot eliminate HIV infection from the general target cells under any circumstances.

Enough scientific information on HIV and AIDS has been gleaned from modern research to warrant comprehensive intervention against the pandemic. Vertical research has revealed many important aspects of the virus and its relationship with the human body, to an extent approaching exhaustion, making it unlikely that we will unravel any more aspects of greater significance in the design of pharmacological or other innovations than have already been achieved. By focusing new research in a different dimension, it is possible to utilize this information to open up new frontiers in the search for a cure for HIV infection. The proven and indisputable scientific information available to advance logic and propositions simply provides a means of convergence of knowledge gathered by documented research, so there is a real prospect of success and no possibility of controversy or scientific conflict. There is instead an opportunity for advancement of knowledge. Providing a backbone of scientific theory can spur specific research that may provide further insight on aspects not considered before, contributing to a generation of new knowledge and development of effective interventions in HIV research.

\section{Acknowledgment}

This paper is a logical analysis of facts that are already scientifically established about HIV and AIDS. Much of the specific information used herein was obtained from various published sources, most of which are referenced. Of particular importance was the use of Internet sources 
to research and obtain these relevant sources. The Google search engine provided valuable links, as well as other websites, such as Wikipedia, private, public, and other accessible domains.

\section{Disclosure}

The author reports no commercial, sectarian, or other interest in the presentation of this material for scientific publication.

\section{References}

1. Weiss RA. How does HIV cause AIDS? Science. 1993;260: 1273-1279.

2. Douek DC, Roederer M, Koup RA. Emerging concepts in the immunopathogenesis of AIDS. Annu Rev Med. 2009;60:471-484.

3. Joint United Nations Programme on HIV/AIDS. Overview of the global AIDS epidemic. UN report on the global AIDS epidemic 2006. Available from: http://data.unaids.org/pub/GlobalReport/2006/2006_ GR_CH02_en.pdf. Accessed November 13, 2011.

4. Joint United Nations Programme on HIV/AIDS. Overview of the global AIDS epidemic. UN report on the global AIDS epidemic 2010. Available from: http://www.unaids.org/globalreport/Global_report.htm. Accessed November 13, 2011.

5. Greener R. AIDS and macroeconomic impact. In: Forsyth S, editor. State of The Art: AIDS and Economics. Available from: http://www. iaen.org/library/statepidemic/chapter7.pdf. Accessed November 13, 2011.

6. Palella FJ Jr, Delaney KM, Moorman AC, et al. Declining morbidity and mortality among patients with advanced human immunodeficiency virus infection. HIV Outpatient Study Investigators. $N$ Engl J Med. 1998;338:853-860.

7. Cunningham AL, Donaghy H, Harman AN, Kim M, Turville SG. Manipulation of dendritic cell function by viruses. Curr Opin Microbiol. 2010;13:524-529.

8. Migueles SA, Connors M. Long-term nonprogressive disease among untreated HIV-infected individuals: clinical implications of understanding immune control of HIV. JAMA. 2010;304:194-201.

9. Lawn SD. AIDS in Africa: the impact of coinfections on the pathogenesis of HIV-1 infection. $J$ Infect. 2004;48:1-12.

10. Buchbinder SP, Katz MH, Hessol NA, O’Malley PM, Holmberg SD. Long-term HIV-1 infection without immunologic progression. AIDS. 1994;8:1123-1128.

11. [No authors listed]. Time from HIV-1 seroconversion to AIDS and death before widespread use of highly active antiretroviral therapy: a collaborative re-analysis. Collaborative Group on AIDS Incubation and HIV Survival including the CASCADE EU Concerted Action. Concerted Action on SeroConversion to AIDS and Death in Europe. Lancet. 2000;355:1131-1137.

12. Morgan D, Mahe C, Mayanja B, Okongo JM, Lubega R, Whitworth JA. HIV-1 infection in rural Africa: is there a difference in median time to AIDS and survival compared with that in industrialized countries. AIDS. 2002;16:597-632.

13. Schneider MF, Gange SJ, Williams CM, et al. Patterns of the hazard of death after AIDS through the evolution of antiretroviral therapy:19842004. AIDS. 2005;19:2009-2018.

14. International Committee on Taxonomy of Viruses 61.0.6. Lentivirus. National Institutes of Health. 2002. Available from: http://www. ncbi.nlm.nih.gov/ICTVdb/ICTVdB/61060000.htm. Accessed November 13, 2011.

15. International Committee on Taxonomy of Viruses.61. Retroviridae. National Institutes of Health. 2002. Available from: http://www. ncbi.nlm.nih.gov/ICTVdb/ICTVdB/61000000.htm. Accessed November 13, 2011.
16. Smith JA, Daniel R. Following the path of the virus: the exploitation of host DNA repair mechanisms by retroviruses. ACS Chem Biol. 2006;1:217-226.

17. Reeves JD, Doms RW. Human immunodeficiency virus type 2. J Gen Virol. 2002;83(Pt 6):1253-1265.

18. Gilbert PB, McKeague IW, Eisen G, et al. Comparison of HIV-1 and HIV-2 infectivity from a prospective cohort study in Senegal. Stat Med. 2003;22:573-593

19. Kahn JO, Walker BD. Acute human immunodeficiency virus type 1 infection. N Engl J Med. 1998;331:33-39.

20. Piatak M Jr, Saag MS, Yang LC, et al. High levels of HIV-1 in plasma during all stages of infection determined by competitive PCR. Science. 1993;259:1749-1754.

21. Pantaleo G, Demarest JF, Schacker T, et al. The qualitative nature of the primary immune response to HIV infection is a prognosticator of disease progression independent of the initial level of plasma viremia. Proc Natl Acad Sci U S A. 1997;94:254-258.

22. Burton GF, Keele BF, Estes JD, Thacker TC, Gartner S. Follicular dendritic cell contributions to HIV pathogenesis. Semin Immunol. 2002;14:275-284.

23. Clapham PR, McKnight A. HIV-1 receptors and cell tropism. Br Med Bull. 2001;58:43-59.

24. Kitahata MM, Gange SJ, Abraham AG, et al. Effect of early versus deferred antiretroviral therapy for HIV on survival. $N$ Engl J Med. 2009;360:1815-1826.

25. Morgan D, Mahe C, Mayanja B, Okongo JM, Lubega R, Whitworth JA. HIV-1 infection in rural Africa: is there a difference in median time to AIDS and survival compared with that in industrialized countries? AIDS. 2002;16:597-632.

26. Grabar S, Selinger-Leneman H, Abgrall S, Pialoux G, Weiss L, Costagliola D. Prevalence and comparative characteristics of long-term nonprogressors and HIV controller patients in the French Hospital Database on HIV. AIDS. 2009;23:1163-1169.

27. Blankson JN. Control of HIV-1 replication in elite suppressors. Discov Med. 2010;9:261-266.

28. Chan DC, Fass D, Berger JM, Kim PS. Core structure of gp41 from the HIV envelope glycoprotein (PDF). Cell. 1997;89:263-273.

29. Levy Y, Lacabaratz C, Weiss L, et al. Enhanced T cell recovery in HIV-1-infected adults through IL-7 treatment. J Clin Invest. 2009; 119:997-1007.

30. Tincati C, d'Arminio Monforte A, Marchetti G. Immunological mechanisms of interleukin-2 (IL-2) treatment in HIV/AIDS disease. Curr Mol Pharmacol. 2009;2:40-45.

31. Schneider MF, Gange SJ, Williams CM, et al. Patterns of the hazard of death after AIDS through the evolution of antiretroviral therapy:19842004. AIDS. 2005;19:2009-2018.

32. HIV vaccine "reduces infection". BBC News. September 24, 2009. Available at: http://news.bbc.co.uk/2/hi/health/8272113.stm. Accessed March 30, 2010.

33. [No authors listed]. A (prime) boost for HIV vaccine research? Lancet. 2009;374:1119.

34. National Institute of Allergy and Infectious Diseases. Treating HIVinfected people with antiretrovirals protects partners from infection. National Institutes of Health News, May 2011. Available from: http:// www.niaid.nih.gov/news/newsreleases/2011/Pages/HPTN052.aspx. Accessed November 12, 2011.

35. Mark Schoofs. A Doctor, a Mutation and a Potential Cure for AIDS. The Wall Street Journal. November 7, 2008. Available from: http:// online.wsj.com/article/SB122602394113507555.html. Accessed November 13, 2011.

36. Hütter G, Nowak D, Mossner M, et al. Long-term control of HIV by CCR5 Delta32/Delta32 stem-cell transplantation. $N$ Engl J Med. 2009;360:692-698. Available from: http://content.nejm.org/cgi/content/ abstract/360/7/692. Accessed November 13, 2011.

37. Allers K, Hutter G, Hofmann J, et al. Evidence for the cure of HIV infection by CCR5 32/32 stem cell transplantation. Blood. 2010; 117:2791-2799. 
38. Coovadia H. Antiretroviral agents - how best to protect infants from HIV and save their mothers from AIDS. $N$ Engl J Med. 2004;351:289-292.

39. Horvath T, Madi BC, Iuppa IM, et al. Interventions for prevention of late postnatal mother-to-child transmission of HIV. Available from: http://www.cochrane.org/reviews/en/ab006734.html. Accessed November 13, 2011.
40. The Joint United Nations Programme on HIV and AIDS. 2009 AIDS Epidemic Update. http://data.unaids.org/pub/Report/2009/JC1700_Epi_ Update_2009_en.pdf. Accessed November 13, 2011.

HIV/AIDS - Research and Palliative Care

\section{Publish your work in this journal}

HIV/AIDS - Research and Palliative Care is an international, peerreviewed open-access journal focusing on advances in research in HIV, its clinical progression and management options including antivira treatment, palliative care and public healthcare policies to contro viral spread. The journal welcomes original research, basic science, clinical \& epidemiological studies, reviews \& evaluations, expert opinion \& commentary, case reports \& extended reports. The manuscript management system is completely online and includes a very quick and fair peer-review system. Visit http://www.dovepress.com/ testimonials.php to read real quotes from published authors.

Submit your manuscript here: http://www.dovepress.com/hivaids---research-and-palliative-care-journal 